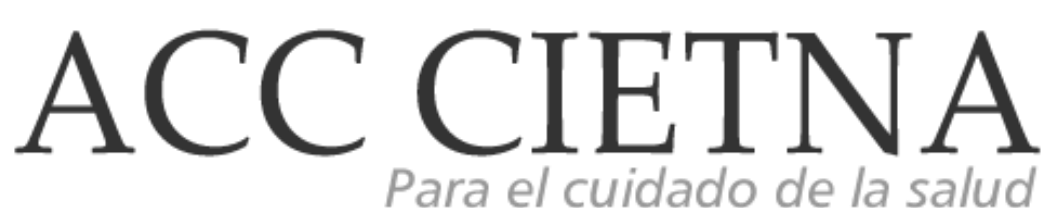

https://doi.org/10.35383/cietna.v3i2.46

\title{
Gestión del conocimiento en los pobladores frente al consumo y calidad del agua en el Caserío Puente Tulipe- Lambayeque.
}

\author{
Coronel Bernilla María Rosalina ${ }^{1}$, Pachamora Díaz Johanna Maribel ${ }^{2}$
}

\begin{tabular}{l} 
INFORMACIÓN DEL ARTÍCULO \\
\hline Historia del artículo: \\
Recibido el 5 de junio de 2016 \\
Aceptado el 5 de octubre de 2016
\end{tabular}

Palabras claves:

Gestión, gestión del conocimiento Agua

Consumo y calidad

Autogestión

Idiosincrasia.

\begin{abstract}
RESUMEN
Este estudio se he enfocó en la problemática de las comunidades de nuestro país en relación al consumo y calidad del agua, siendo éste uno de los factores para afirmar que existe pobreza ultidimensional, reflejando con mayor incidencia en zonas rural - marginales, como es el caso del Caserío Puente Tulipe. Se planteó la siguiente pregunta de investigación: ¿Cómo mejorar la gestión del conocimiento de los pobladores frente al consumo y calidad del agua? El objetivo general es gestionar el conocimiento de los pobladores frente al consumo y calidad del agua. Fue una Investigación Cualitativa con abordaje metodológico de Investigación-acción participativa. El abordaje teórico se basó en el Modelo de Promoción de Salud de Nola J. Pender. Los sujetos de estudio fueron 20 pobladores, quienes habitan el Km32 del canal Taymi. Se utilizó la entrevista semi-estructurada con la técnica de grupos focales. Se respetaron los criterios éticos de Sgreccia y los criterios de rigor científico. Se realizó un análisis temático, obteniendo como resultados: Conocimiento gestionado en los actores sociales frente al consumo y gestión de la calidad del agua y Desarrollo de estilos de vida saludable en la comunidad respecto a calidad, consumo de agua y la repercusión en su salud.
\end{abstract}

\footnotetext{
${ }_{1}^{1}$ Licenciada. Enfermera con trabajo independiente, Chiclayo, Perú. Email: mcoronel@gmail.com

${ }^{2}$ Licenciada. Enfermera con trabajo independiente, Chiclayo, Perú. Email: johani_amen@hotmail.com
} 


\section{Knowledge management in the people against the consumption and} quality of water in the hamlet Bridge Tulipe- Lambayeque.

\section{ABSTRACT}

\section{Keywords:}

Management

Knowledge management

Water

Consumption and quality
The present research proposed a study I focused on the problems of the communities of our country in relation to consumption and water quality, being one of the factors to affirm that there is poverty multidimensional, reflecting a higher incidence in rural areas marginal, a clear example is in the hamlet Tulipe Bridge, where the water supply for the residents is unsafe sources like the way management and consumption, considering the following research question How to improve knowledge management villagers over consumption and water quality?, whose overall objective is to manage the knowledge of the people against the consumption and wáter quality. It was a qualitative research approach Participation Action Research methodology and was implemented in the village TulipePátapo Bridge. The theoretical approach was based on the Health Promotion Model of Nola J. Pender. The study subjects were 20 residents of the hamlet bridge Tulipe, who inhabit the KM32 Taymi channel. To collect the data we used semi-structured interview with the focus group technique. Criteria were respected Sgreccia ethical and scientific criteria. Thematic analysis was performed, obtaining the following results: Knowledge managed social actors in relation to consumption and management of wáter quality and development of healthy lifestyles in the community for quality, water consumption and the impact on their health.

\section{Introducción}

En el mundo no existe otra sustancia de mayor importancia para la humanidad como es el agua, pues constituye las tres cuartas partes de la superficie terrestre. A través del tiempo, su distribución a las poblaciones ha ido comercializándose para poder brindar agua apta para consumo humano, creándose diferentes entidades que tienen como función ofrecer servicios de saneamiento, cuyo producto final es agua de calidad, constituyendo también un medio por el cual se cuida la salud de las personas.

Consumir agua segura es parte del derecho de la persona y obligación del estado el velar por la salud de su país. Son millones de personas que se abastecen de agua que no cumplen las recomendaciones, ni los estándares nacionales e internacionales que se establecen para el consumo humano. ${ }^{1}$ 
Son 70 millones de latinoamericanos que carecen de acceso a agua potable, a pesar que se ha avanzado mucho en términos de cobertura todavía hay áreas grandes de desigualdades, sobre todo en llegar con los servicios a las zonas urbano-marginales y a las rurales, pero el tema no es sólo de cobertura sino de calidad de los servicios; asimismo de los 459 millones de habitantes que hay en América Latina son 95 millones que carecen de saneamiento y 194 millones están conectados a sistemas de alcantarillado cuyos afluentes no reciben tratamiento. ${ }^{2}$

El Perú es uno de los países que tiene el privilegio detener el $1 \%$ del $5 \%$ a nivel mundial de agua dulce, sin embargo es manejado de manera inadecuada, generándose escasez y falta de suministro para aquellas zonas más alejadas y pobres del país. Son 7 millones de peruanos que no están conectados al servicio de agua potable, la respuesta es que el Perú maneja este recurso, cada vez más escaso, de forma ineficiente, sumándole el problema de abastecimiento a las áreas más alejadas sobre todo en zonas rurales donde no hay entidades prestadoras de servicios de saneamiento, menos aún, suministros de agua segura, falta de desagüe y alcantarillado, servicios básicos que toda población debe de satisfacer por derecho, es por eso que los resultados hablan por sí solos al mostrarnos en la Audiencia Pública de la Democracia del Agua: Retos de Futuro, donde coloca al Perú casi en el último lugar en brindar servicios de agua potable y alcantarillado de América Latina. ${ }^{3}$

El agua tiene una repercusión importante dentro del desarrollo social, como un proceso de promoción del bienestar de las personas en conjunción con un proceso dinámico de desarrollo económico, pues en el transcurso del tiempo, conduce al mejoramiento de las condiciones de vida de toda la población en diferentes ámbitos: salud, educación, nutrición, vivienda, vulnerabilidad, seguridad social, empleo, salarios, principalmente implica también la reducción de la pobreza y la desigualdad en el ingreso. En este proceso, es decisivo el papel del estado como promotor y coordinador del mismo, con la activa participación de actores sociales, públicos y privados $.4,5$

La falta de equidad en la distribución del agua, y por ende de saneamiento a los múltiples tipos de abastecimientos que tienen las poblaciones rurales y marginales del Perú, tiene como consecuencia que los pobladores debido a su deficiente conocimiento por el grado de instrucción educativa característico de las zonas rurales, se conviertan en factores determinantes para provocar comportamientos no saludables como el de no clorar el agua, no hervirla antes de consumirla o usarla en preparación de sus alimentos.

El inadecuado consumo provoca riesgos a corto plazo que son el resultado de la contaminación del agua por elementos químicos o microbiológicos que pueden suscitar trastornos en un período que va desde unas pocas horas hasta varias semanas después de la ingesta, originando numerosas enfermedades diarreicas y gastroentéricas. Su número las coloca entre las tres principales causas de muerte en la región latinoamericana, siendo las más comunes: amebiasis, cólera, Hepatitis, Shigelosis, tifoidea, fiebres paratíficas e infecciones por rotavirus. ${ }^{2}$

Además que la forma en cómo la población consume el agua no potable no está ajeno a las realidades a nivel local un claro ejemplo se da en el caserío Puente Tulipe, donde encontramos un problema de salud ambiental referido al tipo de agua para consumo humano, en este caserío existe dos tipos de abastecimiento de agua, el que discurre por el canal Taymi km 32, trayendo muchos contaminante como animales muertos, excretas de animales, basura, etc.; el cual es bombeado a través de un motor, que muchas veces tiene fugas de petróleo que se mezcla con el agua del canal, asimismo este es conducido por tuberías y almacenado en tanque rústicos. La otra manera de extracción es desde el subsuelo con electrobomba y se almacena en un tanque 
elevado en el cual se observa presencia de moho mucilaginoso y formación de una capa blanca.

Asimismo el agua sirve de abastecimiento a las familias, donde se observa la formación de un sedimento blanco, además que al tener contacto con el jabón éste no se disuelve adecuadamente, y cuando es consumida por las personas tiene un sabor de tipo salobre produciendo molestias gastrointestinales después de su ingesta, además que se han reportado 1 caso de hepatitis tipo $B$ y 2 casos de EDAS en el caserío.

Es por eso que se formuló el siguiente problema: ¿Cómo mejorar la gestión del conocimiento de los pobladores frente al consumo y calidad del agua?, cuyo objeto de investigación fue la gestión del conocimiento de los pobladores del Caserío Puente Tulipe, teniendo como objetivo general, mejorar el conocimiento de los pobladores frente al consumo y calidad de agua, y objetivos específicos el poder identificar las capacidades cognitivas de la población sobre el adecuado uso y consumo de agua, analizar, proponer y aplicar gestión del conocimiento en los pobladores frente al consumo y calidad de agua a través de la aplicación de estrategias que permitan desarrollar capacidades cognitivas y comportamientos saludables.

Finalmente la presente investigación estuvo orientada a mejorar el conocimiento en los pobladores frente al consumo y calidad del agua, y lograr desarrollo de capacidades proactivas en búsqueda de su propio bienestar que apunte a una auténtica gestión del conocimiento.

\section{Metodología}

Investigación tipo cualitativa, con abordaje metodológico investigación-acción participativa, pues la persona, familia y comunidad son los principales actores, debido a que no hay quien mejor describa y proponga soluciones al identificar un problema y/o necesidad de salud.
Todo este proceso de investigación se ejecutó en seis fases según la OPS. 6

Fase1: Organización de experiencias y conocimientos previos en el área de estudio.

En esta fase las investigadoras se interesan por temas de desarrollo social, humano y problemas de salud pública, particularmente por el escaso conocimiento de educación sanitaria de poblaciones más vulnerables en frente a la problemática de la calidad y consumo de agua, y buscan que los pobladores logren informarse $y$ formarse al respecto.

Las investigadoras indagaron sobre teorías de salud pública así como en profesionales de Enfermería vinculadas con el primer nivel de atención en salud, salud pública, a los medios de comunicación (internet), a la municipalidad, específicamente el área de saneamiento básico de Pátapo, pues el caserío es de su jurisdicción, además de la información primaria, que fueron los pobladores del caserío, y quienes más conocen de la realidad; por lo que se sintetizó y se recolectó la información para poder develar conocimientos claves e incorporarlos en el proceso de investigación, asimismo esta información contó con un equipo profesional y los actores de cambio. De esta forma, las investigadoras profundizaron la problemática del estudio, la definición clara de términos y la comprensión efectiva y toma de dirección adecuada de la investigación científica.

\section{Fase 2: Definición de la problemática general de la investigación.}

Durante esta fase se tuvo en cuenta el punto de investigación: gestión del conocimiento en los pobladores frente al consumo y calidad del agua, para afianzar y conocer las percepciones, experiencias y lo que piensan acerca del tema por los actores sociales. Para lograrlo se realizó la técnica de los grupos focales o focus group que permitió reunir a los pobladores del caserío 
Puente Tulipe, que viven en el perímetro $\mathrm{Km} 32$ del canal Taymi, además de tener las mismas fuentes de abastecimiento de agua.

Dentro del primer grupo focal o se realizó interrogantes que permitieron ahondar en el tema y conocer la realidad del problema en relación con el conocimiento previo que tienen sobre el consumo y la calidad del agua que utilizan en su vida cotidiana, la importancia que tiene gestionar el conocimiento de los pobladores sobre el tema en cuestión, además de los beneficios que éste traería. Todo ello condujo a la definición conjunta del problema que permitió observar el entendimiento del estudio a realizar por todos los actores sociales.

Siendo el primer procedimiento, realizar la convocatoria, para que los pobladores participen, teniendo como requisitos necesarios el tener que vivir en el Km 32 del caserío Puente Tulipe, puesto que ellos son la base fundamental para conocer la realidad abordada y así poder lograr el cambio que se deseaba; debido a que estos actores sociales habrían de haber experimentado en su cotidianidad esta realidad. Las investigadoras reunieron a los pobladores en un ambiente cálido, tranquilo y de relación interpersonal, promoviendo acogida, incentivándolos a que dieran sus respuestas las más veraces posibles y así ayudar en el análisis e interpretación de los obstáculos o problemas. La entrevista fue grabada y posteriormente analizada.

\section{Fase 3: Objetivación y problematización.}

El problema encontrado y planteado por los pobladores del caserío Puente Tulipe, el área de saneamiento básico de la Municipalidad del distrito de Pátapo fueron: deficiente gestión del conocimiento en los pobladores sobre consumo y calidad del agua, debido al escaso conocimiento que tienen los pobladores sobre la calidad del agua que consumen y la idiosincrasia de la población en sus estilos de vida frente al consumo y calidad del agua y la repercusión en su salud, y con su participación se planteó la priorización de los problemas descubiertos evaluando su viabilidad para el mejoramiento de los problemas priorizados.

\section{Fase 4: Investigación de la realidad social y análisis de la información recolectada.}

En esta fase de define el tema y la pregunta de investigación, que en este estudio es ¿Cómo mejorar la gestión del conocimiento en los pobladores del caserío Puente Tulipe 2011?, luego determinamos que la recolección de datos se realizó con entrevista semiestructurada con pregunta norteadora a profundidad que se ejecutó a través de la técnica de grupos focales, luego recabada la información se realizó el análisis con abordaje de contenido temático, resultando dos categorías y dos subcategorías en cada momento, antes, durante y después de la intervención utilizando los discursos brindados por los pobladores.

\section{Fase 5: Definición de proyectos de acción.}

A través de los problemas planteados fue necesario implementar un programa educativo sobre el consumo y calidad del agua, que permitirá gestionar el conocimiento, generando que los pobladores creen, capturen, transformen y usen los conocimientos, desarrollando así capacidades para buscar soluciones y estrategias en beneficio de su salud, los cuales se identificaron, se analizaron y se comprendieron a través del análisis del contenido tipo temático. Una vez identificados los problemas que obstaculizan la gestión del conocimiento en los pobladores frente al consumo y calidad del agua se organizó, priorizó y planificó cuatro talleres viables que respondían a la problemática encontrada, que fueron denominados: "Agua y desarrollo social", "Introducción sobre consumo y calidad del agua", "Agua en el proceso salud enfermedad" y "Tratamiento, manejo y manipulación del agua”. 
Cada taller constó de una capacitación, en el cual se brindaba la exposición del tema y posteriormente se realizaba una evaluación constante con preguntas a fines a la temática realizada con la finalidad de verificar comprensión y aprendizaje. $\mathrm{Y}$, antes de cada capacitación se les informó a los participantes sobre los temas que se abarcarían en las capacitaciones y el material que se necesitaría para que asistan preparados.

Además teniendo en cuenta que la gestión del conocimiento es necesario trabajar en forma intersectorial se gestionó con la municipalidad (área de saneamiento básico), para la asistencia del encargado, pues se comprometió en apoyar para la elaboración de un proyecto para el mejoramiento del sistema de abastecimiento de agua, asimismo los representantes de la junta administradora de servicio de saneamiento, se comprometieron de forma activa su participación en la limpieza mensual y mantenimiento del tanque elevado, visitas domiciliarias para mejorar la autogestión, vigilancia y control, y a su vez la asamblea general mostró actitud receptiva, satisfecha de los acuerdos tomados, del mismo modo se prometieron confianza mutua, estuvieron activos, interesados y dispuestos a aprender, decir y escuchar, conscientes al cambio; lo cual permitió seguir motivándolos para el logro de los objetivos.

\section{Fase 6: Recuperación y sistematización de la experiencia.}

Se procedió a la recolección de los distintos elementos de la experiencia y desarrollar procesos, resultados e impactos que tendrá la implementación del programa con participación activa de los mismos actores sociales (pobladores, área de saneamiento básico y la Junta de Administradores de servicio de saneamiento del caserío Puente Tulipe, mediante la utilización de un proceso de reflexión colectiva, puesto que al implementar el programa educativo se controló como un proceso de gestión teniendo en cuenta todo el ciclo de planificar, organizar, dirigir y controlar, el cual nos permitió evaluar el impacto en los pobladores frente al consumo y calidad de agua, percibiendo sus experiencias desde el objetivo que se quiere obtener.

Asimismo la sistematización de la experiencia se realizó a través de un proceso de reflexión colectiva, como la convocatoria a una asamblea general con participación de la JASS, Unidad de Saneamiento y agua potable, alcalde y adjuntos, además de la población en general, además de integrar elementos de la teoría que sirvió de marco y de la práctica realizada, reordenándolos y dándoles coherencia, de manera que puedan servir de marco de referencia o guía a otros procesos similares. Asimismo se para recolección de datos se utilizó entrevista semiestructurada con una pregunta norteadora a profundidad, con la técnica de focus group.

Antes de la Intervención: Luego organizar experiencias y conocimientos previos en el área de estudio, definir la problemática, objetivar y problematizar, investigar la realidad social y analizar la información recolectada emergieron dos categorías:

1.- Escaso conocimiento que tienen los pobladores sobre la calidad del agua que consumen.

1.1.- Ausencia de gestión del conocimiento sobre consumo y calidad del agua.

1.2.- Desinterés en la autogestión, vigilancia y control de agua que consumen.

2.- Identificando la idiosincrasia de la población como riesgo en sus estilos de vida frente al consumo y calidad del agua y la repercusión en su salud.

2.1.- Creencias arraigadas sobre consumo de agua y su repercusión en la salud.

2.2.- Prácticas, comportamientos y actitudes inadecuadas en su vida diaria en relación al consumo de agua. 
Durante la Intervención: Después se definió el plan de acción, planificándose actividades los cuales se ejecutaron con participación activa de los actores sociales, es así que emergieron dos categorías durante la intervención:

1.- Mejorando el conocimiento que tienen los pobladores sobre la calidad del agua que consumen.

1.1.- Educando a los pobladores sobre el consumo y calidad de agua.

1. 2. - Fortaleciendo la capacidad de participación

2.- Promocionando la movilización social y desarrollo comunitario para la calidad, consumo del agua y la repercusión en su salud.

2.1.- Adaptando las creencias arraigadas sobre consumo de agua y su repercusión en la salud en una participación activa en su beneficio.

2.2.-Acondicionando las prácticas, comportamientos y actitudes inadecuadas en su vida diaria en relación al consumo de agua segura, en estilos de vida saludable.

Después de la Intervención: En esta etapa de la intervención se recupera y sistematiza la experiencia, para desarrollar los procesos, resultados e impactos de la intervención, surgiendo dos categorías las cuales son:

1.- Conocimiento gestionado en los actores sociales frente al consumo y gestión de la calidad del agua.

1.1.- Conocimiento competente sobre gestión, administración, consumo y calidad de agua.

1.2.-Autogestión, vigilancia y control activa en los actores sociales en el mejoramiento del proceso y sistema de abastecimiento en la población.

2.- Desarrollo de estilos de vida saludable en la comunidad respecto a calidad, consumo de agua y la repercusión en su salud.

2.1.- Desarrollo de nuevos conocimientos y participación activa sobre consumo de agua y su repercusión en la salud.
2.2.- Prácticas, comportamientos y actitudes adecuadas desarrolladas en su vida diaria en relación al consumo de agua.

\section{Análisis y discusión}

\section{Antes de la Intervención}

1.- Escaso conocimiento que tienen los pobladores sobre la calidad del agua que consumen.

1.1.- Ausencia de gestión del conocimiento sobre consumo y calidad del agua.

En esta subcategoría se identifica que no existe gestión del conocimiento sobre consumo y calidad del agua que la población consume, debido a que tanto el proveedor no brinda educación siendo ésta su responsabilidad hacia los integrantes de la Junta Administradora de Servicio y saneamiento (JASS) formada en la Comunidad, y esta a su vez a los pobladores que son los consumidores directos del servicio brindada por la municipalidad y gestionada por la JASS, provocando desinterés, y desconocimiento de la realidad en relación al consumo y calidad del agua que se abastecen, por lo tanto se evidencia en la falta de toma de decisiones, acciones frente a la problemática y la gestión ineficaz que se realiza en conjunto con las autoridades encargadas del servicio de agua, además de prácticas inadecuadas en el manejo dentro de los hogares, es así que se deberá proporcionar a los hogares y a las comunidades, mediante programas de salud con una visión de gestión que tengan como temas ejes: la higiene, los conocimientos necesarios para monitorear y gestionar la inocuidad del agua que consumen. 6

Se identificaron los siguientes discursos:

\section{“...Para consumir el agua la extraemos de los} grifos que tenemos fuera de las casas, la depositamos en nuestros tanques en algunos momentos se hierve para consumirlo o si no la tomamos cruda porque a veces se nos acaba el agua hervida y hasta hervirla demora porque tenemos que pasarla por mantel, pues hay una 
capa blanca que se forma después de hervirla, después la depositamos en una olla o en una jarra y cuando tenemos la necesidad de tomarla cogemos cogemos un vaso y la tomamos o si no de los tanques en los que lo depositamos, los encargados de soltar el agua, a veces se olvidan y tenemos que ir avisarles y encima se molestan, $y$ por eso ni vamos a las reuniones porque vamos a perder el tiempo nada más y eso que pagamos mensualmente una cuota y sólo tenemos una hora para juntar en nuestros baldes...". Clavel.

En los discursos se puede evidenciar que los pobladores tienen escaso conocimiento a consecuencia de la falta de gestión de éste por parte de los encargados y responsables de brindar el servicio de agua potable, pues no brindan un servicio de calidad para que la población consuma agua segura, cuando nos referimos a calidad no sólo se basa en infraestructura, sino también en información y educación el cual se trabaja de una manera intersectorial cuando de saneamiento básico se trata, debido a que constituye parte del desarrollo humano, asimismo cuando hablamos de gestión del conocimiento nos referimos que no sólo se le brinda información sino que los pobladores lo pongan en práctica en su vida cotidiana, a través de la captura efectiva de información, crear soluciones para este problema percibido por ellos mismos como es la falta de gestión de conocimiento en su comunidad, y de esa manera transformar su realidad y usarlo como herramientas que requieran otra situación que puedan afectar su salud.

1.2.- Desinterés en la autogestión, vigilancia y control de agua que consumen.

La vigilancia contribuye a la protección de la salud pública al fomentar la mejora de la calidad, la cantidad, la accesibilidad, la cobertura, la asequibilidad y la continuidad de los sistemas de abastecimiento de agua (conocidos como indicadores de servicio) y se realiza como complemento a la función de control de calidad del proveedor de agua de consumo. La vigilancia del abastecimiento de agua de consumo no elimina ni sustituye la responsabilidad del proveedor, que debe asegurarse de que la calidad del agua de consumo sea aceptable y cumpla las metas de protección de la salud predefinida, así como con otras metas relativas a la eficacia. ${ }^{7}$ Será necesario realizar un seguimiento posterior para asegurarse de que se han aplicado las medidas correctoras. La vigilancia no se limita a los sistemas de abastecimiento de agua de consumo gestionados por un proveedor único, sino que comprende los sistemas gestionados por comunidades e incluye la garantía de una higiene adecuada en la captación y el almacenamiento del agua en los hogares. ${ }^{8}$

Se identificaron los siguientes discursos:

“...Consumir el agua sucia, cae enfermedades, infección, cáncer, además la municipalidad no se preocupa porque el agua esté limpia, ya ni la cloran, ellos deben cuidarlo, pero ya hace varios meses que no le echan lejía, pero si vamos a la municipalidad no nos hacen caso, nosotros trabajamos en la chacra no tenemos tiempo para ver eso del agua, pero hay un vecino que ve las llaves para que nos llegue el agua en las mañanas...". Kentia.

En los diferentes discursos brindados por los pobladores se concluye que los pobladores conocen la problemática de la calidad del agua y la forma inadecuada del consumo que tienen, sin embargo no realizan acciones para poder mejorarlas, justificándose en que el único responsable del problema es la municipalidad, y no crean posibles soluciones para mejorar, mostrando una actitud de desinterés, lo cual se demuestra en la falta de acciones frente a la limpieza del tanque elevado, la coordinación con las diferentes unidades (Unidad de saneamiento y agua potable - municipalidad y saneamiento ambiental - Centro de salud) encargadas para la realización del monitoreo de partículas por millón de cloro residual en el agua del que se abastecen, asimismo muestran dentro de sus debilidades $y$ dificultades la falta de proactividad e iniciativa para generar autogestión, vigilancia comunal y 
monitoreo local para obtener desarrollo comunidad y la salud colectiva.

2.- Identificando la idiosincrasia de la población como riesgo en sus estilos de vida frente al consumo y calidad del agua y la repercusión en su salud.

2.1.- Creencias arraigadas sobre consumo de agua y su repercusión en la salud.

La cultura comprende valores, actitudes, normas, ideas, hábitos y percepciones internalizadas, construyendo las creencias, las cuales se definen como formas o expresiones concretas que se adoptan y que, en gran medida, son compartidas entre un grupo de personas y están en la base de todo comportamiento, individual o colectivo, razón por la cual se deben trabajar en aquellas que son negativas y que puedan representar un riesgo para la salud de la población, y las causas de que sean arraigadas es porque no se han discutido abiertamente, ni durante la socialización, y se van absorbiendo por asimilación y exhibición de conductas inadecuadas.

Es por ello que se debe intervenir y analizar conjuntamente con la comunidad para poder adaptarlas a su realidad y a la vez sentirse beneficiados y satisfechos con la transformación y uso de las creencias saludables en su vida cotidiana y poder generar impactos positivos en su salud y desarrollo. Se identificaron los siguientes discursos:

\section{“...El agua la vemos clara y no tiene mal olor no} puede estar sucia, entonces de que nos vamos a enfermar, además toda agua de tanque está clorada, cuando teníamos el agua del canal, a veces tampoco la hervíamos porque no traía basura ni botaban animales muertos, incluso nos bañábamos con esa agua, pero ahora nos sale ronchas en el cuerpo, y para cocinar usamos el agua del pozo y para lavar usamos la del canal, además aquí la gente no reclama nada, no nos vamos a las reuniones porque los del comité no saben que decirnos, reclamamos y no nos solucionan, y no conocemos como debemos limpiar los pozos, ni como meternos ahí con ellos..." Flor.

De los discursos se puede analizar una creencia arraigada en las personas, acerca de que las enfermedades solo afectan a los niños y no a los adultos, también se puede evidenciar que al prevalecer estas creencias en los pobladores influyen en fomentar una actitud de pasividad, creación de conocimientos inadecuados y por ende conductas y comportamientos desfavorables para su salud, y que al identificar problemas, no actúan, pues reconocen desconocer y sentirse fuera del grupo piloto Junta de Administradores de Saneamiento), el cual es una creencia incorrecta, pues la población es parte de la asamblea general, los cuales son los encargados de generar soluciones en beneficio de la población en relación a saneamiento.

2.2.- Prácticas, comportamientos y actitudes inadecuadas en su vida diaria en relación al consumo de agua.

Las actitudes son variables importantes para poder actuar frente a esta problemática, y las cuales se definen como disposiciones permanentes de ánimo, formadas por un conjunto de convicciones y sentimientos que llevan al sujeto a actuar. Comprenden tres factores: uno ideológico, formado por ideas $y$ convicciones determinadas; otro sentimental que genera simpatía o antipatía hacia cosas o personas; y un tercero reactivo que impulsa a pensar $y$ obrar en las distintas situaciones sociales en consonancia con las convicciones y sentimientos profesados, concretizándose en prácticas y comportamientos de las personas. Se identificaron los siguientes discursos:

“...En la comunidad no sabemos cómo es el cuidado del agua nosotros cargamos el agua hasta nuestros depósitos y cuando se va acabando volvemos a llenarlos, pero también hay personas que no tienen agua en sus casas $y$ toman las aguas que recogen de las tuberías con 
las que riegan las chacras en la parte alta o sino recogen del canal, los del comité se deben preocupar para que todos tengan agua, pero no lo hacen, ellos son los encargados de darnos a todos por igual y más horas, y la gente que botan su basura y nadie dice nada, porque si no dicen que uno es mal vecino ya mejor para evitarnos problemas con los demás no decimos nada..." Narciso.

Dentro de las manifestaciones se evidencia como actitud inadecuada a la pasividad pues al detectar problemas y necesidades de salud no les dan importancia y no la socializan para buscar solución y trabajar en ellos, además que muestran conformidad la cual en esta situación es negativa pues la población modifica sus sentimientos, opiniones y conductas en dirección a la posición mantenida por el grupo mayoritario, como resultado de la pasividad y falta de participación social.

\section{Durante la Intervención:}

1.- Mejorando el conocimiento que tienen los pobladores sobre la calidad del agua que consumen.

1.1.- Educando a los pobladores sobre el consumo y calidad de agua.

La estrategia idónea para desarrollar y potenciar el capital humano más importante, el conocimiento, es la educación sanitaria la cual es un proceso dirigido a promover estilos de vida saludables (hábitos, costumbres, comportamientos) a partir de las necesidades específicas del individuo, familia o comunidad. Desde este punto de vista, la educación sanitaria comprende un conjunto de actividades educativas desarrolladas en procesos formales e informales, que ejecutan permanentemente (educación continua) todos los actores, como parte de las actividades institucionales; no se limita a la transmisión puntual de mensajes mediante talleres educativos o demostraciones. ${ }^{8}$
Se identificaron los siguientes discursos:

“...Nosotros no tenemos agua dentro de nuestras casas sacamos agua a veces del canal y si hay agua del tanque, y a veces si nos enseñan no sabemos que el agua del tanque pueda estar contaminada si no le echan cloro y que puede enfermarnos a todos, por eso el agua debe estar limpia, para estar sanos todos, además es en beneficio de todos aquí, porque así como nos han dicho, el agua es un beneficio para nuestra sociedad, nuestra salud más que todo y ya pues si no nos enfermamos, no vamos a gastar en medicinas..." Clavel

En los discursos podemos evidenciar que los pobladores reconocen que los talleres educativos han contribuido a la construcción de un conocimiento para mejorar su salud, la de su familia y comunidad, mencionan prácticas importantes dentro del consumo cómo el de manejo, manipulación adecuada y el aspecto de calidad, el uso de cloro y la vigilancia, autogestión y control de la JASS, asimismo manifiesta que ellos también son responsables de la salud en la comunidad y la importancia que se les eduque en temas de salud, puesto que es un factor importante dentro de la calidad de vida $y$ económicamente.

2.2.- Fortaleciendo la capacidad de participación de los actores sociales en el mejoramiento de la autogestión, vigilancia y control de agua que consumen. La participación real de la comunidad es importantísima, tanto para poder identificar sus problemas, como para solucionarlos. Cuando hablamos de participación real, nos referimos a la toma de decisiones. La participación de la ciudadanía en todas las etapas de los programas de calidad del agua es una de las estrategias indispensables para que esos programas tengan éxito e impacto. El principal actor del proceso de promoción de la calidad del agua es la población, la cual se debe constituir en objeto y sujeto del proceso. ${ }^{10}$ 
Además, la propia comunidad es la que mejor conoce su situación y las causas que las provocan. $Y$ es también la más interesada, en buscar y encontrar las soluciones. Nadie, desde afuera, puede imponer cosas que las personas de la comunidad, no acepten ni compartan ${ }^{11}$.

A través del fortalecimiento tanto de la JASS, pues es una de las organizaciones más importantes dentro de la comunidad pues administra, opera y mantienen los servicios de saneamiento, en este caso el servicio de agua segura a la comunidad así como también la municipalidad, la unidad de servicio de agua potable, ente encargado de brindar seguridad a la población. Se identificaron los siguientes discursos:

“...Hemos estado trabajando para obtener un mejoramiento en el sistema de agua, por eso ahora estamos empadronando a los pobladores para saber cuántos somos, y enviar una propuesta al alcalde, además esta capacitación nos ha servido para saber cuáles son nuestra funciones como comité, $y$ ahora como ya sabemos vamos a poner todo de nuestra parte, , $y$ como presidente de la JASS, tengo como función representar a nuestros usuarios, y como también hemos escuchado por la capacitación que no estamos solos, sino también vamos a trabajar con la municipalidad y el centro de salud, son muchas cosas que nos faltan pero es nuestro compromiso con nuestra población vigilar que el servicio de agua sea de calidad, porque les afecta en su salud y también en sus cultivos..."

Girasol.

En los discursos se puede evidenciar que tienen muy en claro a los actores sociales en la comunidad cuál es el papel de la JASS, la municipalidad y un área específica como es la unidad de servicio de agua potable, asimismo de los pobladores mismos, lo importante es que reconocen su deber como comunidad en su desarrollo, y la necesidad por tener un sistema para todos, con mejor servicio y seguridad para su salud, además señalan propuestas para el mejoramiento y ampliación señalando un compromiso con su objetivo, finalmente se puede señalar que el fortalecimiento permite que cada actor social participe dentro de sus funciones $y$ niveles de cargo, si trabajan conjuntamente pueden consolidar alianzas permitiendo un beneficio para la comunidad.

2.- Promocionando la movilización social y desarrollo comunitario para la calidad, consumo del agua y la repercusión en su salud.

2.1.-Adaptando las creencias arraigadas sobre consumo de agua y su repercusión en la salud en una participación activa en su beneficio.

La adaptación se define como la superación de una realidad y la posibilidad de una mayor calidad de vida, bienestar, desarrollo económico y social de un grupo de personas en un lugar común, es así que temas tan importantes como la salud y agua tienen connotaciones culturales, pues, la cultura puede incidir de forma positiva o negativa en el bienestar individual, es por eso que las prácticas prácticas en la gestión de los recursos hídricos deberían adaptarse a las distintas culturas, pues cada una de el las constituye un sistema particular de conocimientos. Del mismo modo, la cultura ha de tenerse plenamente en cuenta en las estrategias de gestión de los recursos hídricos; La diversidad cultural es una fuente de prácticas sostenibles y enfoques innovadores ${ }^{12}$.

El desarrollo comunitario representa la superación de una realidad y la posibilidad de una mayor calidad de vida, bienestar, desarrollo económico y social de un grupo de personas en un lugar común. La participación es un eje central de la intervención comunitaria, A través de ella se busca promover, favorecer y dinamizar la participación de la comunidad en todo el proceso de desarrollo de los programas de intervención. Sin esta participación será difícil lograr una intervención que responda a las necesidades de la comunidad y que mejore su calidad de vida ${ }^{13}$. Se identificaron los siguientes discursos: 
“...bueno yo uso el agua para tomar cruda sin cocinar y ahora sé que debo hervirla, antes de tomarla y ponerlo en un deposito donde no se contamine $y$ así no podamos enfermar, también ahora sé que el agua que viene del pozo no está limpia como para consumirla porque no se clora, y ahora sé que debo clorarla si no puedo hervirla..." Flor.

En los discursos se pueden identificar como los pobladores tienen creencias adquiridas por su propia cultura, por conocimientos que son trasmitidos de generación en generación; pero a través de la educación sanitaria adecuada son capaces de comprender y comparar lo que conocen con lo que van aprendiendo.

2.2.- Acondicionando las prácticas, comportamientos y actitudes inadecuadas en su vida diaria en relación al consumo de agua segura, en estilos de vida saludable.

La población de puente Tulipe siempre ha sido abastecida por agua no apta para consumo humano puesto que era la única fuente de abastecimiento y la falta de conocimiento de la población hizo que estas a través de los años se acostumbraran a consumirla inadecuadamente sin las medidas de limpieza y desinfección normadas, posteriormente la forma de abastecimiento actual (fuente de abastecimiento del subsuelo), sigue teniendo la misma forma de uso. En la población hay conductas que constituyen una amenaza para la conservación de la calidad del agua. El cambio de comportamiento en higiene requiere que los materiales sean apropiados al contexto social y cultural en el que se utilizarán y que los educadores conozcan y se adapten ${ }^{12}$. Se identificaron los siguientes discursos:

“...nosotros no sabíamos que si no cuidamos el agua ya no habría agua para tomar, y tampoco que si no hervimos el agua bien nos podemos enfermar, yo no tenía la costumbre de lavar bien los depósitos pero con esto que nos enseñan ya lo voy hacer también voy a cuidar mejor en como uso el agua tanto para tomar y para asearnos todos los que viven en mi casa..." Narciso.

En los discursos podemos identificar que los pobladores a través del conocimiento que se les brinda mejoran sus comportamientos en beneficio de su salud, puesto que identifican la importancia de adecuar $\mathrm{y} / \mathrm{o}$ mejorar los conocimientos que tienen como parte de sus prácticas diarias como es el uso de materiales como baldes, botellas entre otros con fines secundarios como es el uso de baldes de aceite en depósitos de agua con una adecuada limpieza y de manera adecuada, conjuntamente con los nuevos conocimientos que van adquiriendo para mejorar sus estilos de vida en beneficio de su salud. Asimismo se pueden identificar como las personas del sexo femenino por estar en mayor contacto con la manipulación, tratamiento del agua y uso; son las más interesadas en mejorar sus estilos de vida con la finalidad de dar un ambiente saludable para su familia.

\section{Después de la Intervención:}

1.- Conocimiento gestionado en los actores sociales frente al consumo y gestión de la calidad del agua.

1.1.- Conocimiento competente sobre gestión, administración, consumo y calidad de agua. Según la real academia define competente como, pericia, aptitud, idoneidad para hacer algo o intervenir en un asunto determinado. Es decir un conocimiento competente es aquel que se se ajusta a la realidad, es oportuno, y convenientemente se ha desarrollado después de adquirir nuevos conocimientos. Es así que se evidencia que la educación para la salud con visión de gestión en el conocimiento influye en todos los resultados de desarrollo humano. Promueve el desarrollo y la economía local, debido a que las enfermedades de transmisión por diferentes reservorios, como el agua, los alimentos contaminados, dejan de ser el primer problema sanitario de un país y ceden su lugar a las enfermedades crónicas y más aún lograr el desarrollo humano y disminuir la pobreza, pues 
en la actualidad la pobreza se define no sólo como sinónimo de bajo nivel de ingresos, sino también de debilitamiento de toda una serie de capacidades humanas fundamentales, incluidas las relacionadas con la salud ${ }^{14}$.

Se identificaron los siguientes discursos:

“...Hemos aprendido que el agua segura es la que no te da enfermedades, que conforme vamos cuidando en nuestra casa nos va ayudar para no enfermarnos, cosa que también ahorramos, nosotros ya estamos comprometidos con los de la junta porque es un bien para todos mensualmente vamos a realizar el lavado del tanque, y también ir a las asambleas, cui dar el agua que consumimos es importante porque es vida, y también la utilizamos para nuestras tierras, animales por eso tenemos que cuidarla todos aquí en el caserío..." Clavel.

En los discursos se evidencia que los pobladores han concientizado la problemática que en algún momento enfrentaron, $y$ al tener la oportunidad de ser educados en relación al consumo y calidad del agua buscaron beneficiarse para mejorar y fortalecer la autogestión, la capacidad de participación, generar conocimientos nuevos de tácitos hacia explícitos (gestión del conocimiento), adecuar costumbres, prácticas y comportamientos hacia unos saludables, promocionar movilización social y desarrollo comunitario, asimismo reconocen la importancia de los talleres educativos, mencionan aspectos importantes como agua segura, calidad, e interrelacionar el término agua con economía, desarrollo y participación social, lo cual eran los objetivos del estudio, mejorar el conocimiento de los pobladores, el cual paralelamente se logra la reducción de tasas de mortalidad por EDAS, enfermedades gastrointestinales 14

En el Caserío Puente Tulipe la población expresa que las EDAS han reducido, puesto que en la actualidad el agua que consumen es segura, debido a que se ha iniciado control y vigilancia en el sistema de abastecimiento (tanque de agua), asimismo dentro sus domicilios la higiene ha mejorado considerablemente.
1.2.- Autogestión, vigilancia y control activa en los actores sociales en el mejoramiento del proceso y sistema de abastecimiento en la población.

La vigilancia del consumo y calidad del agua es el conjunto de acciones adoptadas por la propia comunidad que en coordinación con la autoridad sanitaria competente, que en este estudio sería el centro de salud en coordinación con la Dirección Regional de Salud.

Por lo tanto trabajar y capacitar desde un inicio o en el transcurso de su ejecución como autoridad local se convierte en una estrategia crucial para que el sistema de abastecimiento, y/o servicio que se brinda sea eficiente y la comunidad tenga la seguridad de que el agua que consume sea de calidad, constituyéndose así la autogestión donde la autoridad local y comunidad busca el trabajo intersectorial iniciando una retroalimentación entre autoridades competentes. Se identificaron los siguientes discursos:

“...Hemos aprendido bastante y también agradecemos la iniciativa de ustedes porque a veces en nuestro comité teníamos problemas porque teníamos que solucionarlo todo nosotros, porque íbamos a cada rato y nos decían esperen, pero ahora sabemos que tenemos derechos $y$ bueno también deberes que nos respaldan ante cualquier injusticia, ahora ya somos ciudadanos porque participamos en nuestro desarrollo y bueno a través de estas capacitaciones hemos ido mejorando, por ejemplo en darnos ideas de cómo trabajar en conjunto, ahora vamos a empezar para hacer nuestro proyecto en conjunto con la unidad de servicio de agua potable de la municipalidad, y también vamos a ver más sobre la vigilancia, el control de la calidad del agua en conjunto con el centro de salud para las muestras, estas cosas son muy importantes para nosotros, para nuestro desarrollo..." Coriandro.

Los pobladores reconocen la importancia que tienen desarrollar capacidades las que le permite generar habilidades en los aspectos de su función como autoridad local, como Junta Administradora 
de Servicio de Saneamiento, y también tienen en cuenta que hay un trabajo en conjunto con las autoridades regionales, y se sienten satisfechos, puesto que tienen la percepción que no están abandonados, sino que toman en cuenta sus opiniones, asimismo cuando mencionan al agua lo hacen como un elemento que promueve el desarrollo social y humano de la comunidad, debido a que el agua promueve o desincentiva el crecimiento económico y el desarrollo social de una comunidad.

2.- Desarrollo de estilos de vida saludable en la comunidad respecto a calidad, consumo de agua y la repercusión en su salud. .

2.1.-Desarrollo de nuevos conocimientos y participación activa sobre consumo de agua y su repercusión en la salud.

Es necesario superar las concepciones tradicionales de desarrollo, donde las comunidades participaban como mano de obra, para disminuir costos. La participación comunitaria se debe dar en el analizar, el hacer y el decidir. Por lo tanto, las actividades incluyen acciones de información, educación, consulta, fortalecimiento de la iniciativa, fiscalización, concertación, toma de decisiones y gestión. ${ }^{15}$ Se identificaron los siguientes discursos:

“...señorita hemos aprendido a que nosotros debemos participar en manejar bien los sistemas de agua para toda la comunidad, porque esto es de todos nosotros, estamos trabajando en la ampliación del sistema de agua con una institución privada ojala se acepte el proyecto que la municipalidad nos está ayudando también, en mi familia ahora sabemos que es un derecho tener agua limpia y de calidad; y que eso ayuda a que se desarrolle la comunidad cuando usamos adecuadamente el agua ya sea cuando la consumimos o cuando la usamos para bañarnos o lavar ropa..." kentia.

En los discursos se evidencia que la población a través de la educación sanitaria impartida logra la capacidad de crear nuevo conocimiento el cual al aplicarse constantemente se han convertido en actitudes y costumbres saludables evidenciándose en sus actividades diarias a través del compromiso que ha asumido la población en la práctica de acciones que brinden salud a su familia y comunidad, generando empoderamiento por su parte permitiéndoles analizar los factores que los afecta, lograr el desarrollo social y económico de la comunidad, a través de un trabajo conjunto de manera solidaria, colaborativa, con las destrezas necesarias para adoptar estilos de vida saludables que se hacen notables.

2.2.- Prácticas, comportamientos y actitudes adecuadas desarrolladas en su vida diaria en relación al consumo de agua.

Las creencias que la población tiene sobre el agua producto del acoplamiento de conocimientos trasmitidos de generación en generación, limitan el proceso de apertura al nuevo conocimiento, es por ello que la educación sanitaria como parte de la estrategia de promoción de la salud busca desarrollar y fortalecer capacidades y actitudes para que la población asuma la importancia en el bienestar de su salud la práctica de comportamientos y actitudes en su vida diaria, con ello disminuirá la proporción de enfermedades y mejora el desarrollo de la comunidad en los aspectos sociales, económicos y culturales en bienestar de la salud de la comunidad.

Se identificaron los siguientes discursos:

“...bueno ahora yo he aprendido que el adecuado manejo del agua en casa disminuye las enfermedades, lo fuimos aprendiendo a través de la educación que nos dieron para tomar un mayor y mejor conocimiento de sistema de agua y de la forma como se abastece a toda la comunidad, asimismo del manejo que toda la comunidad debe conocer sobre la distribución del agua y la importancia de la participación en el cuidado..." Narciso. 
En los discursos se evidencia la práctica de comportamientos y actitudes adecuadas desarrolladas diariamente por la población en relación al consumo de agua segura, puesto que reconocen que muchos problemas de salud como parasitosis, infecciones intestinales, etc. no son producto de creencias y costumbres sino de malas prácticas de higiene en el hogar, desde el manejo extra domiciliario hasta el almacenamiento intra domiciliario y uso.

El compromiso de la población del cuidado de los sistemas de agua, las fuentes abastecedoras de agua para la comunidad y la formación de un comité para vigilancia y control del suministro de agua.

\section{Conclusiones}

En la actualidad se enfatiza el nivel estructural para determinar que un servicio es de calidad; sin embargo, todo el proceso no es evaluado, creando problemas de sostenibilidad de los proyectos de abastecimiento de agua, razón por el cual, la gestión del conocimiento tanto a nivel técnico conformado por la Junta de Administración de Servicios y Saneamiento y pobladores que conforman la asamblea general constituye una herramienta importantísima, porque permite desarrollar capacidades $y$ habilidades cognitivas, administradoras y de gestión, comportamientos saludables, transferencia de conocimiento, logrando la participación social activa, convertirse de poblador a ciudadano, capaz de identificar necesidades y problemas en su comunidad, para luego buscar soluciones sostenibles y viables en beneficios de todos, esta visión se logra a través de la ejecución de las diferentes estrategias de promoción de la salud, pues busca generar mecanismos administrativos, organizativos $y$ políticos que faciliten tener mayor control sobre su salud y condición de vida.
Por lo tanto, los resultados evidencian que la gestión del conocimiento es necesaria y fundamental para crear sostenibilidad en proyectos no sólo de agua, sino de salud, puesto que, el recurso fundamental es el conocimiento, capital humano por excelencia, capaz de ser desarrollado y mejorado, transfiriéndolo del conocimiento tácito hacia uno explicito para permitir el cambio de un paradigma asistencial y paternal hacia uno participativo de actores sociales interesados en fomentar factores favorables para su salud y desarrollo humano.

\section{Bibliografía}

1. Organización panamericana de la salud, Organización Mundial de la Salud. Agua y salud. División de Salud y Ambiente, 1999.

2. Ercilio M, cabelm I. Desafíos del derecho humano al agua en el Perú. Segunda edición: 1000 ejemplares; editorial: gráfica loro'ss.a.lima : 2005.

3. Midgley J. Social Development: The Developmental Persjpective in Social Welfare, Londres, Sage, 1995.

4. Sen A. Desarrollo y Libertad, Editorial Planeta, México, 2000.

5. Kozier B, Erb G. "Fundamentos de Enf e r me $r$ í a : Conc e pt os, Pr oc e s o Y Práctica" España: Mc Graw - Hill Interamericana, 1999.

6. Organización Panamericana de la Salud. Planificación local participativa: Metodologías para la promoción de la salud en América Latina y el Caribe Canada: OPS, 1999.

7. OPS, OMS. Guía de orientación en saneamiento básico para alcaldías de municipios rurales y pequeñas comunidades. 2009. 
8. Real Academia Española. Disponible en url: http://www.rae.es/rae.html

9. Fernández B. Guía de promoción y desarrollo comunitario para asegurar la calidad del agua en los países en desarrollo.

Editorial, Centro Panamericano de Ingeniería Sanitaria y Ciencias del Ambiente, Lima: Perú, 2004

10. Organización Panamericana de la Salud.

Agua y saneamiento: Evidencias para políticas públicas con enfoque en derechos humanos $y$ resultados en salud pública. 25 Twenty-third Street, N.W. Washington, D.C. 20037, E.U.A. 2011.

11. Giraldo B, OPS/CEPIS/PUB.04.104. Guía de promoción y desarrollo comunitario para asegurar la calidad del agua en los países en desarrollo. Lima- Perú. 2014

12. Manual de Educación Sanitaria: Saneamiento Básico Rural ministerio de salud Cajamarca, 2008.

13. CARE -PERU. El componente de la educación sanitaria en proyectos de agua $y$ saneamiento. 2011

14. OPS/OMS. Orientaciones Sobre Agua Y Saneamiento Para Zonas Rurales. 2008

15. CARE -PERU. Guía para la organización y trabajo de la JASS. $2^{\text {a }}$ ed. 2002 